\title{
On the Ratio of Inverted Gamma Variates
}

\author{
M. Masoom $\mathrm{Ali}^{1}$, Manisha $\mathrm{Pal}^{2}$, and Jungsoo $\mathrm{Woo}^{3}$ \\ Department of Mathematical Sciences, Ball State University, USA \\ Department of Statistics, University of Calcutta, India \\ Department of Statistics, Yeungnam University Gyongsan, South Korea
}

\begin{abstract}
In this paper the distribution and moments of the ratio of independent inverted gamma variates have been considered. Unbiased estimators of the parameter involved in the distribution have been proposed. As a particular case, the ratio of independent Levy variates have been studied.
\end{abstract}

Zusammenfassung: In diesem Aufsatz werden die Verteilung und Momente vom Quotient unabhängiger invertierter Gammavariablen betrachtet. Unverzerrte Schätzer für den Parameter in der Verteilung werden vorgeschlagen. Als ein spezieller Fall wird der Quotient unabhängiger Levy-Variablen untersucht.

Keywords: Moments, Unbiased Estimator, Maximum Likelihood Estimator, Levy Variables.

\section{Introduction}

The distribution of the ratio of random variables are of interest in problems in biological and physical sciences, econometrics, classification, and ranking and selection. Examples of the use of the ratio of random variables include Mendelian inheritance ratios in genetics, mass to energy ratios in nuclear physics, target to control precipitation in meteorology, and inventory ratios in economics. The distribution of ratio of random variables have been studied by several authors like Marsaglia (1965) and Korhonen and Narula (1989) for normal family, Press (1969) for student's t family, Basu and Lochner (1979) for Weibull family, Provost (1989) for gamma family, Pham-Gia (2000) for beta family, among others. The distribution of the ratio of independent gamma variates with shape parameters equal to 1 was studied by Bowman, Shenton, and Gailey (1998). Recently, Ali, Woo, and $\mathrm{Pal}$ (2006) obtained the distribution of the ratio of generalized uniform variates.

In this paper we derive the distribution of the ratio $V=X /(X+Y)$, where $X$ and $Y$ are independent inverted gamma variates, each with two parameters. An inverted gamma distribution $\mathrm{IG}(p, \sigma)$ is given by

$$
f(x ; p, \sigma)=\frac{\sigma^{p}}{\Gamma(p)} x^{-p-1} e^{-\sigma / x}, \quad x>0, \sigma>0, p>0,
$$

where $p$ is the shape parameter and $\sigma$ the scale parameter.

The moments of the distribution of the ratio have been obtained. As a particular case, the ratio of independent Levy variables has been considered. The Levy distribution is one of the few distributions that are stable and that have probability density functions that are analytically expressible. Moments of the Levy distribution do not exist. But the distribution is found to be very useful in analysis of stock prices and also in Physics for the study of dielectric susceptibility (see Jurlewicz and Weron, 1993). 


\section{Distribution of the Ratio of Inverted Gamma Variables}

Let $X$ and $Y$ be independent random variables distributed as $\operatorname{IG}\left(p, \sigma_{x}\right)$ and $\operatorname{IG}\left(q, \sigma_{y}\right)$, respectively. Then $U=1 / X$ and $W=1 / Y$ are independently distributed as $\operatorname{Gamma}\left(p, \sigma_{x}\right)$ and $\operatorname{Gamma}\left(q, \sigma_{y}\right)$, respectively. We note that $V=X /(X+Y)=W /(U+W)$. Let $T=U+W$. Then $V$ and $T$ are jointly distributed with pdf

$$
f_{V, T}(v, t)=\frac{\sigma_{x}^{p} \sigma_{y}^{q}}{\Gamma(p) \Gamma(q)} e^{-t\left\{\sigma_{x}+\left(\sigma_{y}-\sigma_{x}\right) v\right\}} t^{p+q-1} v^{q-1}(1-v)^{p-1}, \quad 0<v<1, t>0 .
$$

Hence the marginal pdf of $V$ is given by

$$
f_{V}(v)=\frac{v^{q-1}(1-v)^{p-1}}{\rho^{q} B(q, p)}\left(1+\frac{1-\rho}{\rho} v\right)^{-p-q}, \quad 0<v<1, \rho=\frac{\sigma_{x}}{\sigma_{y}}>0 .
$$

After some algebraic manipulation, and using formula 8.391 in Gradshteyn and Ryhzik (1965), the cumulative distribution function (cdf) of $V$ is obtained as

$$
F_{V}(v)=\frac{\{1+\rho(1-v) / v\}^{-q}}{q B(q, p)}{ }_{2} F_{1}\left(q, 1-p ; q+1 ;\{1+\rho(1-v) / v\}^{-1}\right), \quad 0<v<1,
$$

where

$$
{ }_{2} F_{1}(a, b ; c ; x)=\sum_{i=0}^{\infty} \frac{(a)_{i}(b)_{i}}{(c)_{i} i !} x^{i}, \quad(a)_{i}=a \cdot(a+1) \cdot \ldots \cdot(a+i-1), \quad(a)_{0}=1,
$$

is the Gauss hypergeometric series.

Using formula 3.197(3) in Gradshteyn and Ryhzik (1965), formulas 15.3.3 and 15.3.5 in Abramowitz and Stegtun (1970), and the density (1), we obtain the moments of the ratio $V=X /(X+Y)$ as

$$
\mathrm{E}\left(V^{k}\right)= \begin{cases}\frac{B(q+k, p)}{B(q, p)}{ }_{2} F_{1}(k, p ; p+q+k ;(\rho-1) / \rho), & \text { if } \rho>1, \\ \rho^{k} \frac{B(q+k, p)}{B(q, p)}{ }_{2} F_{1}(k, q+k ; p+q+k ; 1-\rho), & \text { if } 0<\rho<1 .\end{cases}
$$

In order to estimate $\rho$, we make use of the following lemma.

Lemma 2.1: Let $R=V /(1-V)$. Then

(a) $R$ is distributed as the ratio of two independent random variables with distributions

$\operatorname{Gamma}\left(q, \sigma_{y}\right)$ and $\operatorname{Gamma}\left(p, \sigma_{x}\right)$.

(b) $\mathrm{E}\left(R^{k}\right)=\rho^{k} B(q+k, p-k) / B(q, p)$, provided $p>k$.

Proof: We have $R=V /(1-V)=X / Y=Y^{-1} / X^{-1}$. Since $X$ and $Y$ are independently distributed as $\operatorname{IG}\left(p, \sigma_{x}\right)$ and $\operatorname{IG}\left(q, \sigma_{y}\right)$, respectively, (a) easily follows.

The distribution of $R$ is therefore defined by the pdf

$$
f_{R}(r)=\frac{1}{\rho^{q} B(q, p)} \frac{r^{q-1}}{(1+r / \rho)^{p+q}}, \quad r>0 .
$$


Hence, the $k$-th moment of $R$ comes out to be

$$
\mathrm{E}\left(R^{k}\right)=\frac{B(q+k, p-k)}{B(q, p)} \rho^{k}, \quad \text { provided } p>k .
$$

From the lemma, for $p>1$, we have

$$
\mathrm{E}(R)=\mathrm{E}\left(\frac{V}{1-V}\right)=\frac{q}{p-1} \rho
$$

so that $\mathrm{E}(R) \cdot(p-1) / q=\rho$. Thus, for a random sample $V_{1}, \ldots, V_{n}$ of size $n$ from the distribution of $V$, an unbiased estimator of $\rho$ will be given by

$$
\hat{\rho}=\frac{p-1}{n q} \sum_{i=1}^{n} \frac{V_{i}}{1-V_{i}}, \quad \text { if } p>1 \text {. }
$$

The variance of this estimator is

$$
\operatorname{var}(\hat{\rho})=\frac{p+q-1}{n q(p-2)} \rho^{2}, \quad \text { for } p>2 .
$$

On the basis of independent random samples $X_{1}, \ldots, X_{n_{1}}$ and $Y_{1}, \ldots, Y_{n_{2}}$ drawn from the distributions of $X$ and $Y$, respectively, the maximum likelihood estimator (MLE) of $\rho$ is $\tilde{\rho}=\tilde{\sigma}_{x} / \tilde{\sigma}_{y}$, where $\tilde{\sigma}_{x}$ and $\tilde{\sigma}_{y}$ are the MLEs of $\sigma_{x}$ and $\sigma_{y}$ given by

$$
\tilde{\sigma}_{x}=\frac{n_{1} p}{\sum_{i=1}^{n_{1}}\left(1 / X_{i}\right)}, \quad \tilde{\sigma}_{y}=\frac{n_{2} q}{\sum_{i=1}^{n_{2}}\left(1 / Y_{i}\right)}
$$

Noting that $U=\sum_{i=1}^{n_{1}}\left(1 / X_{i}\right)$ and $W=\sum_{i=1}^{n_{2}}\left(1 / Y_{i}\right)$ are independently distributed as $\operatorname{Gamma}\left(n_{1} p, \sigma_{x}\right)$ and $\operatorname{Gamma}\left(n_{2} q, \sigma_{y}\right)$, respectively, $Z=(1 / U) /(1 / U+1 / W)$ is distributed with pdf given by (1) where $p$ and $q$ are replaced by $n_{1} p$ and $n_{2} q$, respectively. Also,

$$
\tilde{\rho}=\frac{n_{1} p}{n_{2} q} \frac{Z}{1-Z}
$$

such that, from (3),

$$
\mathrm{E}(\tilde{\rho})=\frac{n_{1} p}{n_{1} p-1} \rho
$$

Hence,

$$
\tilde{\tilde{\rho}}=\frac{n_{1} p-1}{n_{1} p} \tilde{\rho}
$$

is an unbiased estimator of $\rho$ with

$$
\operatorname{var}(\tilde{\tilde{\rho}})=\frac{n_{1} p+n_{2} q-1}{n_{2} q\left(n_{1} p-2\right)} \rho^{2} .
$$

It can be easily seen that for $n_{1}=n_{2}=n$ we get $\operatorname{var}(\hat{\rho})>\operatorname{var}(\tilde{\tilde{\rho}})$. 


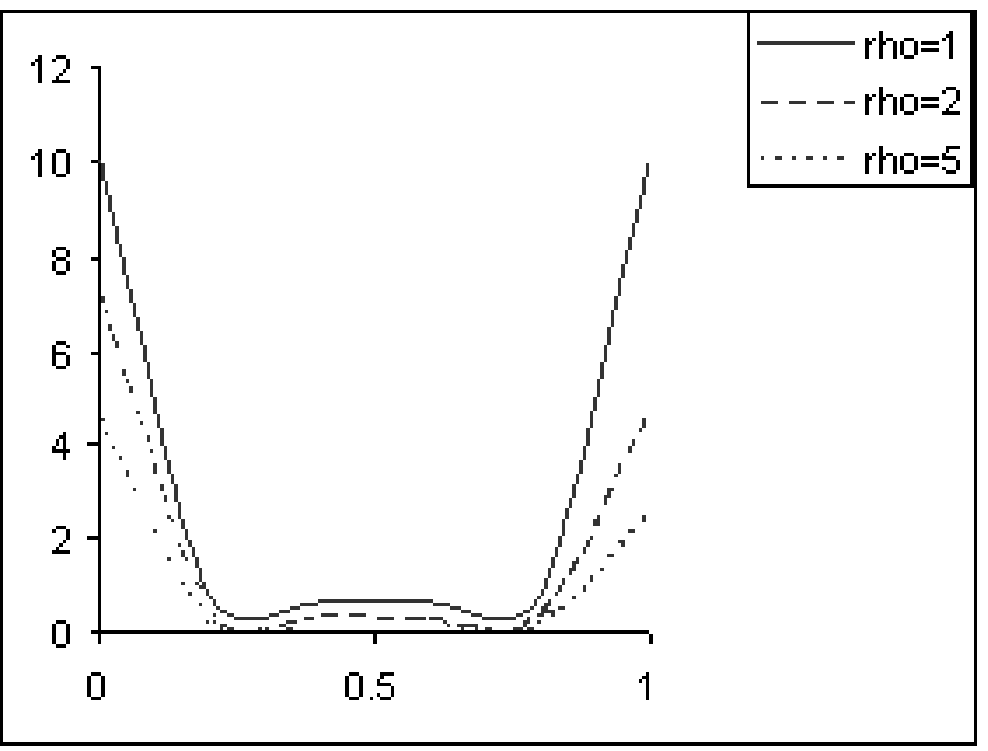

Figure 1: Plots of the pdf (6) for $\rho=1,2,5$.

\section{Distribution of the Ratio of Levy Variables}

For $p=q=1 / 2, X$ and $Y$ are two independent Levy variables with scale parameters $\sigma_{x}$ and $\sigma_{y}$, respectively. The pdf and cdf of $V$ then reduces to

$$
\begin{aligned}
f_{V}(v) & =\frac{1}{\pi \sqrt{\rho}} v^{-1 / 2}(1-v)^{-1 / 2}\left(1+\frac{1-\rho}{\rho} v\right)^{-1}, \\
& =\frac{\sqrt{\rho}}{\pi} v^{-3 / 2}(1-v)^{-1 / 2}\left(1+\rho \frac{1-v}{v}\right)^{-1}, \quad 0<v<1, \rho=\frac{\sigma_{x}}{\sigma_{y}}>0
\end{aligned}
$$

and

$$
\begin{aligned}
F_{V}(v) & =\frac{2}{\pi}\left(1+\rho \frac{1-v}{v}\right)^{-1 / 2}{ }_{2} F_{1}\left(1 / 2,1 / 2 ; 3 / 2 ;(1+\rho(1-v) / v)^{-1}\right) \\
& =\frac{2 \sqrt{\rho}}{\pi} \sqrt{\frac{1-v}{v}\left(1+\rho \frac{1-v}{v}\right)^{-1}{ }_{2} F_{1}\left(1,1 ; 3 / 2 ;(1+\rho(1-v) / v)^{-1}\right)} \\
& =\frac{2}{\pi} \sin ^{-1} \frac{1}{\sqrt{1+\rho \frac{1-v}{v}}}, \quad 0<v<1 .
\end{aligned}
$$

The expression (7) of the cdf is obtained using formulas 3.381(3) and 6.455(1) in Gradshteyn and Ryhzik (1965), and expression (8) follows from formula 15.1.6 in Abramowitz and Stegtun (1970).

From (2) the moments of the distribution are

$$
\mathrm{E}\left(V^{k}\right)= \begin{cases}\frac{B(k+1 / 2,1 / 2)}{\pi}{ }_{2} F_{1}(k, 1 / 2 ; 1+k ;(\rho-1) / \rho) & \text { if } \rho>1 \\ \frac{\rho^{k} B(k+1 / 2,1 / 2)}{\pi}{ }_{2} F_{1}(k, k+1 / 2 ; 1+k ; 1-\rho) & \text { if } 0<\rho<1 .\end{cases}
$$


From Abramowitz and Stegtun (1970), we have the following useful relations for the hypergeometric function ${ }_{2} F_{1}(a, b ; c ; z)$ :

\section{Lemma 3.1:}

(i) (Formula 15.2.2)

$$
\frac{\partial^{n}}{\partial z^{n}}{ }_{2} F_{1}(a, b ; c ; z)=\frac{(a)_{n}(b)_{n}}{(c)_{n}}{ }_{2} F_{1}(a+n, b+n ; c+n ; z)
$$

(ii) (Formula 15.1.13)

$$
{ }_{2} F_{1}(a, a+1 / 2 ; 1+2 a ; z)=\frac{2^{2 a}}{(1+\sqrt{1-z})^{2 a}}
$$

(iii) (Formula 15.1.14)

$$
{ }_{2} F_{1}(a, a+1 / 2 ; 2 a ; z)=\frac{2^{2 a-1}}{\sqrt{1-z}(1+\sqrt{1-z})^{2 a-1}}
$$

(iv) (Formula 15.3.5)

$$
{ }_{2} F_{1}(a, b ; c ; z)=(1-z)_{2}^{-b} F_{1}(b, c-a ; c ; z /(z-1)) .
$$

From Lemma 3.1 we get the following lemmas:

\section{Lemma 3.2:}

(a)

$$
{ }_{2} F_{1}(1,3 / 2 ; 2 ; z)=\frac{2}{\sqrt{1-z}(1+\sqrt{(1-z)})}
$$

(b)

$$
{ }_{2} F_{1}(2,5 / 2,3 ; z)=\frac{4}{3} \frac{1+2 \sqrt{1-z}}{(1-z)^{3 / 2}(1+\sqrt{1-z})^{2}} .
$$

Proof: (a) follows from Lemma 3.1(iii) by substituting $a=1$. By Lemma 3.1(i) we get

$$
{ }_{2} F_{1}(2,5 / 2 ; 3 ; z)=\frac{4}{3} \frac{\partial}{\partial z}{ }_{2} F_{1}(1,3 / 2 ; 2 ; z) .
$$

Hence, using (a), we have (b).

\section{Lemma 3.3:}

(a)

$$
{ }_{2} F_{1}(1 / 2,1 ; 2 ;(\rho-1) / \rho)=\frac{2 \sqrt{\rho}}{1+\sqrt{\rho}}
$$


(b)

$$
{ }_{2} F_{1}(1 / 2,2 ; 3 ;(\rho-1) / \rho)=\rho_{2}^{2} F_{1}(2,5 / 2 ; 3 ; 1-\rho)=\frac{4}{3} \frac{\sqrt{\rho}(1+2 \sqrt{\rho})}{(1+\sqrt{\rho})^{2}} .
$$

Proof: (a) follows from Lemma 3.1(ii) by substituting $a=1 / 2, z=(\rho-1) / \rho$, and (b) follows from Lemma 3.1(iv) and Lemma 3.2(b) by taking $a=1 / 2, b=2, c=3$, and $z=(\rho-1) / \rho$.

Using Lemmas 3.2 and 3.3 we have

$$
\mathrm{E}(V)=\frac{\sqrt{\rho}}{1+\sqrt{\rho}}, \quad \mathrm{E}\left(V^{2}\right)=\frac{\sqrt{\rho}(1+2 \sqrt{\rho})}{2(1+\sqrt{\rho})^{2}}
$$

such that

$$
\operatorname{var}(V)=\frac{\sqrt{\rho}}{2(1+\sqrt{\rho})^{2}}
$$

If $\tilde{\rho}$ denotes the MLE of $\rho$ based on independent random samples $X_{1}, \ldots, X_{n_{1}}$ and $Y_{1}, \ldots, Y_{n_{2}}$ from the distributions of $X$ and $Y$, then, from (4) and (5), an unbiased estimator of $\rho$ and its variance are given by

$$
\begin{gathered}
\tilde{\tilde{\rho}}=\frac{n_{1}-2}{n_{1}} \tilde{\rho} \\
\operatorname{var}(\tilde{\tilde{\rho}})=\left(\frac{n_{1}-2}{n_{1}}\right)^{2} \frac{2\left(n_{1}+n_{2}-2\right)}{n_{2}\left(n_{1}-4\right)} \rho^{2}, \quad \text { for } n_{1}>4 .
\end{gathered}
$$

Since $\rho^{*}=\sqrt{\rho} /(1+\sqrt{\rho})$ is a monotone increasing and bounded function of $\rho$, inference on $\rho^{*}$ will be equivalent to inference on $\rho$. Hence, from any estimator of $\rho^{*}$ we can obtain an estimator of $\rho$ by a one-to-one transformation.

From (9) and (10), for a random sample $V_{1}, \ldots, V_{n}$ of size $n$ from the distribution (6), an unbiased estimator of $\rho^{*}$ is $\bar{V}=n^{-1} \sum_{i=1}^{n} V_{i}$ with variance $\rho^{*}\left(1-\rho^{*}\right) / 2 n$. The corresponding estimator of $\rho$ is $\hat{\hat{\rho}}=(\bar{V} /(1-\bar{V}))^{2}$ with asymptotic variance $2 \rho^{3 / 2}(1+$ $\sqrt{\rho})^{2} / n$.

\section{Acknowledgement}

The authors thank the referee for his/her suggestions, which immensely helped to improve the presentation of the paper.

\section{References}

Abramowitz, M., and Stegtun, I. A. (1970). Handbook of Mathematical Functions. New York: Dover Publication Inc.

Ali, M. M., Woo, J., and Pal, M. (2006). Distribution of the ratio of generalized uniform variates. Pakistan Journal of Statistics, 22, 11-19.

Basu, A. P., and Lochner, R. H. (1979). On the distribution of the generalized life distributions. Technometrics, 33, 281-287. 
Bowman, K. O., Shenton, L. R., and Gailey, P. C. (1998). Distribution of the ratio of gamma variates. Communications in Statistics - Simulation and Computation, 27, $1-19$.

Gradshteyn, I. S., and Ryhzik, I. M. (1965). Tables of Integrals, Series and Products. New York: Academic Press.

Jurlewicz, A., and Weron, K. A. (1993). Relationship between asymmetric Levy-stable distributions and the dielectric susceptibility. Journal of Statistical Physics, 73, 69-81.

Korhonen, P. J., and Narula, S. C. (1989). The probability distribution of the ratio of the absolute values of two normal variables. Journal of Statistical Computation and Simulation, 33, 173-182.

Marsaglia, G. (1965). Ratios of normal variables and ratios of sums of uniform variables. Journal of the American Statistical Association, 60, 193-204.

Pham-Gia, T. (2000). Distributions of the ratios of independent beta variables and applications. Communications in Statistics - Theory and Methods, 29, 2693-2715.

Press, S. J. (1969). The t ratio distribution. Journal of the American Statistical Association, 64, 242-252.

Provost, S. B. (1989). On the distribution of the ratio of powers of sums of gamma random variables. Pakistan Journal of Statistics, 5, 157-174.

Authors' addresses:

M. Masoom Ali

Department of Mathematical Sciences

Ball State University

Muncie, IN 47306

USA

E-mail: mali@bsu.edu

Manisha Pal

Department of Statistics

University of Calcutta

35 Ballygunge Circular Road

Kolkata - 700019

India

E-mail: manishapal2@gmail.com

Jungsoo Woo

Department of Statistics

Yeungnam University Gyongsan

South Korea 